\title{
Comportamento do gramado submetido aos herbicidas clethodim e sethoxydim ${ }^{(1)}$
}

\author{
CLEBER DANIEL de G. MACIEL ${ }^{(2)}$; JULIANA P. POLETINE ${ }^{(2)}$; EMANUEL de L. SOUZA ${ }^{(3)}$; \\ LEANDRO de S. ALVES ${ }^{(3)}$, RODRIGO B. RIBEIRO ${ }^{(3)}$ e MICHEL A. RAIMONDI ${ }^{(3)}$ \\ RESUMO
}

\begin{abstract}
O principal fator do custo de manutenção de um gramado é o corte na altura adequada. O crescimento vegetativo e reprodutivo do gramado formado com grama-batatais (Paspalum notatum Flügge), submetido às doses de clethodim e sethoxydim foi avaliado em dois experimentos conduzidos no campus urbano da Escola Superior de Agronomia de Paraguaçu Paulista-ESAPP (SP), em gramado já estabelecido. Foi utilizado o delineamento experimental de blocos casualizados com seis tratamentos e quatro repetições, representados por clethodim nas subdoses de 0,$0000 ; 0,0037$; 0,$0075 ; 0,0150 ; 0,0300$ e $0,0600 \mathrm{~kg}$ i.a. ha ${ }^{-1}$, assim como pelo sethoxydim a 0,$0000 ; 0,0156 ; 0,0312 ; 0,0625 ; 0,1250$ e $0,2500 \mathrm{~kg}$ i.a. ha ${ }^{-1}$. O clethodim e sethoxydim, aos 7 DAA, intoxicaram as plantas a partir das doses de 0,0300 e $0,1250 \mathrm{~kg} \mathrm{ha}^{-1}$, respectivamente. Os sintomas de intoxicação, aos 49 DAA, praticamente desapareceram para todos os tratamentos. O teor de clorofila nas folhas não apresentou diferenças significativas entre as subdoses de clethodim e sethoxydim em relação à testemunha, em nenhuma das épocas estudadas. Houve redução progressiva da altura média e no número de inflorescências do gramado em função do incremento das doses de clethodim e sethoxydim. Estes herbicidas apresentam viabilidade na redução vegetativa e reprodutiva da espécie $P$. notatum, sendo o sethoxydim mais seletivo em relação à preservação das características visuais do gramado.
\end{abstract}

Palavras-chave: Paspalum notatum, gramado, herbicidas, crescimento.

\section{ABSTRACT \\ Behavior of turfgrass treated with clethodim and sethoxydim herbicides}

\begin{abstract}
The main factor of turfgrass maintenance cost is mowing to the appropriate height. Turfgrass (Paspalum notatum Flügge) vegetative and reproductive growth as affected by applications of clethodim and sethoxydim was evaluated in two experiments conducted at Escola Superior de Agronomia de Paraguaçu Paulista, urban campus, at Paraguaçu Paulista Municipal District, São Paulo State, in an area where turfgrass is already established. A randomized complete block experimental design was used with six treatments and four repetitions, represented by clethodim at 0,0000 ;

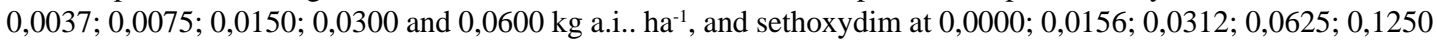
and $0,2500 \mathrm{~kg}$ a.i. ha ${ }^{-1}$. Seven days after application, Clethodim and sethoxydim, caused toxicity to the plants starting from 0,0300 and $0,1250 \mathrm{~kg} \cdot \mathrm{ha}^{-1}$, respectively. Forty-nine days after application, toxicity symptoms practically disappeared for all of the treatments. Chlorophyll content in the leaves did not show significant differences between clethodim and sethoxydim treatments in relation to the check, in none of the periods analyzed. There was a progressive reduction of medium height and number of turfgrass inflorescences in conjunction with clethodim and sethoxydim concentrations. These herbicides presented viable options to reduce vegetative and reproductive growth of P. notatum, with sethoxydim maintaining the better visual characteristics compared to treatments with clethodim.
\end{abstract}

Key words: Bahiagrass, turfgrass, herbicides, growth.

\section{INTRODUÇÃO}

A espécie Paspalum notatum Flügge, também conhecida como grama-batatais, grama-matogrosso, grama-forquilha e grama-bahia, entre outros nomes, é proveniente das Américas Central e do Sul, sendo predominante na região centro-sul do Brasil (ALCÂNTRA e BUSFARAH, 1979; DEMATTÊ, 1983; KISSMANN, 1997). São plantas herbáceas perenes, rizomatozas, de 15 a $30 \mathrm{~cm}$ de altura, com folhas de $10-15 \mathrm{~cm}$ de comprimento, lanceoladas, alongadas e pilosas, concentradas na parte basal, suas inflorescências geralmente são formadas por dois racemos opostos, como "forquilhas".
Os gramados são pouco pesquisados no Brasil, resultando na escassez de informações técnicas e importação das mesmas e/ou generalização de soluções e recomendações sem base científica (BARBOSA et al., 1997).

No Brasil, a espécie $P$. notatum é a grama mais disseminada (ALCÂNTRAe BUSFARAH, 1979; LOPES, 1981). Além de ornamentar jardins, seu gramado pode servir para formar pastagens, cobrir campos de futebol, atuar na estabilização de encostas e controle da erosão, entre outras finalidades, assim como também se tornar daninha em pastagens e em algumas culturas perenes (ANGELIS NETO e ANGELIS, 1999; LORENZI, 2000). Apesar da sua fácil adaptação a solos de baixa fertilidade, condições hídricas

\footnotetext{
(1) Recebido para publicação em 06/03/2006 e aceito em 10/10/2007.

(2) Prof. Dr. Departamento de Ciências Biológicas, ESAPP, 19700-000, Paraguaçu Paulista (SP).

(3) Aluno do Curso de Agronomia, ESAPP, 19700-000, Paraguaçu Paulista (SP).
} 
precárias e pisoteio, o seu rápido desenvolvimento exige cortes freqüentes para a manutenção da qualidade do gramado (GOATLEY et al., 1998)

Pesquisas desenvolvidas nos Estados Unidos comprovam muitos benefícios dos gramados ao ambiente, destacando ajuste natural, confortável e seguro para divertimento e prática de esportes; liberar oxigênio; refrigerar o ar; controlar a poluição e reduzir a erosão do solo; purificar e reter a água dos lençóis freáticos; satisfazer o ser humano pela beleza da paisagem, além de valorizar o imóvel (TURFGRASS PRODUCERS INTERNATIONAL, 2002).

Os gramados podem ser utilizados em diversos locais com os diferentes propósitos, sendo que nas mais diversas situações o principal fator do custo de manutenção é o corte na altura adequada (RODRIGUES et al., 2004). Nesse sentido, a utilização de novas técnicas alternativas, como a aplicação de reguladores vegetais, pode possibilitar a redução dos custos de manutenção de gramados. Além disso, segundo ITOGRASS (2003) e UNRUH (2004), o corte das folhas dos gramados por procedimento de poda pode reduzir sua tolerância ao estresse ambiental, sendo que os principais efeitos são perda de água, desenvolvimento de doenças, diminuição no armazenamento de carboidratos, aumento da intensidade de brotação e diminuição do crescimento das raízes e rizomas.

Os reguladores vegetais estão ganhando maior importância nos programas de manejo de gramados (RICHARDSON, 2002). Embora originalmente introduzidos para reduzir a freqüência do número de podas e a redução do florescimento (JOHNSON, 1990; 1994), estes promovem também maior tolerância dos gramados à seca (JIANG e FRY, 1998) e a sombra (QUIAN e ENGELKE, 1999).

Alguns herbicidas graminicidas aplicados em pós-emergência também podem ser utilizados em subdoses reduzidas para inibir o desenvolvimento e crescimento (WATSHCKE et al., 1977; FREITAS et al., 1997), pela interrupção da síntese de lipídios das plantas, assim como ser bastante efetivos na redução do florescimento. Entretanto, o principal fator limitante desses compostos está na estreita faixa de tolerância aos herbicidas e cuidados com a aplicação, pois doses acima do limite podem causar intoxicação, levando o gramado à morte (KAUFMANN, 1986).

O presente trabalho teve como objetivo avaliar o comportamento do gramado formado com grama-batatais submetido aos herbicidas clethodim e sethoxydim.

\section{MATERIALEMÉTODOS}

O trabalho foi constituído por dois experimentos conduzidos no período de novembro de 2003 a março de 2004, em gramado já estabelecido de grama-batatais do campus urbano da Escola Superior de Agronomia de Paraguaçu Paulista - ESAPP, localizado no Médio Vale do Paranapanema, com altitude média de $480 \mathrm{~m}$, latitude $22^{\circ}$ 24' 46" S e longitude 50³4'33"'W (IBGE-CIDADES, 2004), sendo o clima Aw, classificado por Koeppen, ou seja, clima de savana com inverno seco.

Os experimentos foram constituídos por seis tratamentos e quatro repetições, nos quais foram avaliados os efeitos de cinco subdoses dos herbicidas clethodim e sethoxydim sobre o desenvolvimento vegetativo e reprodutivo do gramado. O delineamento experimental utilizado, em ambos experimentos, foi o de blocos casualizados com quatro repetições e unidades experimentais representadas por parcelas com área de $1,0 \mathrm{x}$ $3,0 \mathrm{~m}\left(3,0 \mathrm{~m}^{2}\right)$. Na Tabela 1 encontram-se representadas as respectivas dosagens dos tratamentos estudados nos experimentos 1 e 2 .

As aplicações dos tratamentos foram efetuadas no final da tarde, em 22/11/2003, utilizando-se pulverizador costal à base de $\mathrm{CO}_{2}$, com pontas DG 110.02, pressão de $2,1 \mathrm{kgf} . \mathrm{cm}^{-2}$ e consumo de calda de $200 \mathrm{~L}^{\mathrm{h}} \mathrm{ha}^{-1}$. No momento das aplicações o solo encontrava-se parcialmente úmido, temperatura do ar de $27,3 \pm 2{ }^{\circ} \mathrm{C}$, umidade relativa $67 \pm 3 \%$ e velocidade dos ventos de $1,4 \pm 5 \mathrm{~km} \mathrm{~h}^{-1}$. Durante a aplicação dos tratamentos, as parcelas foram protegidas lateralmente com lona plástica, para se evitar deriva da pulverização para as parcelas laterais.

Tabela 1. Representação das dosagens utilizadas como tratamentos nos experimentos 1 e 2. ESAPP, Paraguaçu Paulista (SP), 2003/ 2004.

Table 1. Representation of dosages used as treatments in experiments 1 and 2.

\begin{tabular}{lccc}
\hline Tratamentos & $\begin{array}{c}\text { Dosagem Herbicida } \\
\left(\mathrm{kg} \mathrm{i.a.} \mathrm{ha} \mathrm{h}^{-1}\right.\end{array}$ & $\begin{array}{c}\text { Dosagem Herbicida } \\
\left(\mathrm{mL} \mathrm{pc} \mathrm{ha}^{-1}\right)\end{array}$ & $\begin{array}{c}\text { Dosagem Adjuvante* } \\
(\% \text { volume/volume })\end{array}$ \\
\hline Experimento 1 & & & $0,25 \%$ \\
T1. clethodim** & 0,0600 & 250,00 & $0,25 \%$ \\
T2. clethodim & 0,0300 & 125,00 & $0,25 \%$ \\
T3. clethodim & 0,0150 & 62,50 & $0,25 \%$ \\
T4. clethodim & 0,0075 & 31,25 & $0,25 \%$ \\
T5. clethodim & 0,0037 & 15,62 & - \\
T6. testemunha & - & - & $0,25 \%$ \\
Experimento 2 & & & $0,25 \%$ \\
T1. sethoxydim*** & 0,2500 & 250,00 & $0,25 \%$ \\
T2. sethoxydim & 0,1250 & 125,00 & $0,25 \%$ \\
T3. sethoxydim & 0,0625 & 62,50 & $0,25 \%$ \\
T4. sethoxydim & 0,0312 & 31,25 & - \\
T5. sethoxydim & 0,0156 & 15,62 & \\
T6. testemunha & - & - &
\end{tabular}

Obs: $*$ Nome comercial $=$ Aterbane BR ${ }^{\circledR} ; * *$ Nome comercial $=$ Select ${ }^{\circledR} ; * * *$ Nome comercial $=$ Poast $^{\circledR} ;$

P.S.: ${ }^{*}$ Commercial name $=$ Aterbane $\mathrm{BR}^{\circledR} ; *{ }^{*}$ Commercial name $=$ Select $^{\circledR} ; * * *$ Commercial name $^{*}$ Poast $^{\circledR}$; 
Os dados meteorológicos referentes às médias semanais de temperatura $\left({ }^{\circ} \mathrm{C}\right)$ máxima e mínima, pluviometria $(\mathrm{mm})$ e umidade relativa do ar $(\%)$ podem ser observados na Tabela 2.

Aos 7, 21, 35 e 49 dias após a aplicação dos tratamentos (DAA), foram avaliadas as seguintes características: porcentagem de intoxicação da parte aérea do gramado, conforme escala percentual de notas visuais, em que "0\%" correspondeu a "ausência de intoxicação" e "100\%" à "morte total" do gramado, proposta por E.W.R.C. (1964) e SBCPD (1995); teor de clorofila da parte mediana das folhas utilizando-se clorofilômetro portátil, modelo SPAD-502 (Minolta ${ }^{\mathrm{TM}}$ ); altura média do gramado, obtida pela distância vertical entre a superfície do solo e ponta das folhas em sua inclinação natural, utilizando-se amostragens aleatórias; e aos 80 DAA o número de inflorescências. ${ }^{-2}$, com auxílio de quadrado vazado com dimensões de $0,5 \times 0,5 \mathrm{~m}$.

Os dados obtidos foram submetidos à análise de variância pelo teste $\mathrm{F}$ e as suas médias comparadas pelo teste Tukey ao nível de $5 \%$ de probabilidade. Para as características altura e número de inflorescência foram estabelecidas curvas de regressão, conforme recomendações de PIMENTEL GOMES (1987) e COUSENS (1988).

\section{RESULTADOSE DISCUSSÃO}

Todas as doses de clethodim promoveram sintomas de intoxicação visíveis à parte aérea do gramado a partir dos 7 DAA, destacando-se os maiores danos para 0,0300 e $0,0600 \mathrm{~kg} \mathrm{ha}^{-1}$ (Tabela 3), assim como os menores efeitos para $0,0037 \mathrm{~kg} \mathrm{ha}^{-1}$, onde as injúrias foram praticamente ausentes, não diferindo da testemunha sem herbicida. A partir dos 21 DAA, apenas as doses 0,0075 e $0,0037 \mathrm{~kg} \mathrm{ha}^{-1}$ ainda preservavam baixo nível de intoxicação do gramado, mas sendo semelhantes à testemunha. Aos 49 DAA observou-se que clethodim em todos os tratamentos, representava apenas 4,5 e 3,3\% de intoxicação nas pontas e bordas das folhas do gramado para doses 0,0300 e $0,0600 \mathrm{~kg} \mathrm{ha}^{-1}$, respectivamente. FREITAS et al. (1997) observaram sintomas semelhantes para o clethodim em 0,0360 e 0,0480 $\mathrm{kg} \mathrm{ha}^{-1}$, e a recuperação das plantas intoxicadas ocorreu entre 15 a 30 DAA.

O sethoxydim (Tabela 4) foi menos prejudicial à parte aérea do gramado quando comparado aos efeitos promovidos pelo clethodim, embora os sintomas visuais de intoxicação fossem muito semelhantes. As doses de 0,0625; 0,0312 e 0,0156 $\mathrm{kg} \mathrm{ha}^{-1}$ tiveram elevada seletividade à grama-batatais, proporcionando apenas leves injúrias nas pontas e bordas de algumas folhas até aos 21 DAA $(=3,0 \%)$, tendo esses sintomas persistido até os 49 DAA.

Os sintomas de intoxicação no gramado foram caracterizados pela coloração vermelho-amarelada irregularmente distribuídas nas pontas e bordas em direção á base das folhas, progredindo para morte plena das estruturas assemelhando-se a "palitos enegrecidos". Em função desse aspecto, constatou-se que as maiores doses promoveram um aspecto de "raleamento químico" do dossel, a partir da morte plena de várias lâminas foliares, mas sem prejudicar a integridade vegetativa total do gramado. Esse efeito ainda precisa de maiores estudos e considerações, mas inicialmente sugere, para situações de adensamento elevado de folhas e em que a qualidade visual não é prioridade, uma possível redução da "parte aérea verde", referente ao dossel dos gramados de $P$. notatum, com a aplicação em pós-emergência de doses de herbicidas exclusivamente graminicidas, tais como o clethodim e sethoxydim.

Com relação ao teor médio de clorofila das folhas do gramado, não foram constatadas diferenças significativas entre as doses dos herbicidas e o tratamento testemunha (Tabelas 5 e 6) em nenhuma das épocas estudadas. Entretanto, vale a ressalva de que essa avaliação foi efetuada com auxílio de um clorofilômetro portátil, sendo na amostragem padronizado o posicionado da fotocélula do equipamento na porção mediana das folhas do gramado, onde os sintomas de intoxicação não foram tão expressivos quanto nas pontas e bordas das folhas. Os herbicidas clethodim e sethoxydim são classificados como o mesmo mecanismo de ação por atuarem paralisando a síntese de

Tabela 2. Médias semanais de temperatura, pluviometria e umidade relativa do ar (U.R.), registradas durante desenvolvimento dos experimentos.

Table 2. Weekly averages of temperature, rains and relative humidity of air (U.R.), registered during development of the experiments.

\begin{tabular}{|c|c|c|c|c|}
\hline \multirow[t]{2}{*}{ Semanas } & \multicolumn{2}{|c|}{ Temperaturas $\left({ }^{\circ} \mathrm{C}\right)$} & \multirow{2}{*}{$\begin{array}{c}\text { Pluviometria } \\
(\mathrm{mm})\end{array}$} & \multirow{2}{*}{$\frac{\text { U. R. do ar }}{(\%)}$} \\
\hline & Mínimas & Máximas & & \\
\hline $3^{\mathrm{a} / N o v \_2003}$ & 17 & 28 & 11 & 61 \\
\hline $4^{\mathrm{a} / N o v \_2003}$ & 18 & 30 & 59 & 65 \\
\hline 1ªtDez_2003 & 19 & 29 & 118 & 76 \\
\hline 2a/Dez_2003 & 18 & 30 & 18 & 66 \\
\hline 3ª̈Dez_2003 & 19 & 30 & 0 & 60 \\
\hline 4ª̈/Dez_2003 & 19 & 29 & 31 & 58 \\
\hline $1^{\mathrm{a} / \mathrm{J} a n \_2004}$ & 19 & 25 & 30 & 64 \\
\hline $2^{\mathrm{a} / J}$ Jan_2004 & 21 & 30 & 3 & 75 \\
\hline $3^{\mathrm{a} / J a n \_2004}$ & 19 & 31 & 5 & 58 \\
\hline $4^{\mathrm{a} / J}$ an_2004 & 19 & 28 & 40 & 71 \\
\hline 1ª /Fev_2004 & 19 & 31 & 24 & 70 \\
\hline 2a/Fev_2004 & 17 & 27 & 39 & 60 \\
\hline
\end{tabular}


lipídios a partir inibição da enzima Acetil coenzima-A Carboxilase (ACCase) nos cloroplastos das plantas (WSSA, 1994; RODRIGUES e ALMEIDA, 2005; VIDALe MEROTTO JÚNIOR, 2001) com sintomas visíveis de intoxicação semelhantes.

$\mathrm{Na}$ Figura 1, encontram-se dispostas as equações de regressão ajustadas para característica altura do gramado. Os dados originais referentes as diferentes doses de clethodim ajustaram-se de forma satisfatória aos modelos polinomial quadrático $\left(\mathrm{R}^{2}=0,93\right)$, polinomial cúbico $\left(R^{2}=0,99\right)$ e linear $\left(R^{2}=0,89\right)$, respectivamente, para as avaliações realizadas aos 7 DAA, 21 DAA e 35 DAA e 49 DAA. Para o sethoxydim, os efeitos das subdoses sobre a característica altura do gramado ajustaram-se ao modelo de regressão linear aos $7 \mathrm{DAA}\left(\mathrm{R}^{2}\right.$ $=0,71), 21$ DAA $\left(R^{2}=0,84\right), 35$ DAA $\left(R^{2}=0,90\right)$ e 49 DAA $\left(\mathrm{R}^{2}=0,68\right)$. De forma geral, os resultados indicam ter ocorrido redução progressiva na altura média do gramado em função do incremento das subdoses dos herbicidas clethodim e sethoxydim. Entretanto, é importante ressaltar que o aumento da concentração também favoreceu a ocorrência de maior intoxicação na parte aérea do gramado de $P$. notatum, as quais caracterizaram-se no trabalho por persistirem até 35 e/ou 49 DAA.

Ainda com relação a altura do gramado, na Figura 2 observa-se que aos 49 DAA os tratamentos com as doses 0,0300 e $0,0600 \mathrm{~kg} \mathrm{ha}^{-1}$ de clethodim proporcionaram reduções percentuais da ordem de $7,8 \% \mathrm{e}$ $13,2 \%$, respectivamente, podendo ser uma opção interessante para ampliar-se o intervalo de corte em áreas onde a qualidade visual do gramado de $P$. notatum não compromete sua característica de utilização. Nesse contexto, considerando que o intervalo total de avaliação do tempo de crescimento do gramado foi de 49 dias para as subdoses dos herbicidas e a testemunha sem aplicação, para o clethodim poder-se-ia considerar possível uma redução estimada no intervalo de corte em aproximadamente 18, 10 e 6 dias para doses de 0,0600; 0,0300 e $0,0150 / 0,0075 / 0,0037 \mathrm{~kg} \mathrm{ha}^{-1}$, respectivamente. Para o sethoxydim, as subdoses de 0,1250 e $0,2500 \mathrm{~kg} \mathrm{ha}^{-1}$ reduziram o crescimento do gramado aos 49 DAA em torno de $6,6 \%$ e $10,5 \%$ (Figura 2), respectivamente, mas diferente do clethodim, caracterizaram-se por apresentar baixa intoxicação no gramado a partir dos 35 DAA. A redução estimada no intervalo de corte para o sethoxydim foi de aproximadamente 14,9 e 8 dias para doses de 0,$2500 ; 0,1250$ e 0,$0625 ; 0,0312 ; 0,0156 \mathrm{~kg} \mathrm{ha}^{-1}$, respectivamente.

Com relação ao número de inflorescências por unidade de área aos 80 DAA (Figura 3), os dados originais referentes as doses dos herbicidas clethodim e sethoxydim ajustaram-se aos modelos de regressão polinomial quadrático $\left(R^{2}=0,79\right)$ e linear $\left(R^{2}=0,78\right)$, respectivamente. As estimativas dos modelos indicaram que todas as doses estudadas reduziram o número de inflorescências por unidade de área no gramado, corroborando com os resultados relatados por FREITAS et al. (1997). A redução do número de inflorescências apresentou-se progressiva em função do aumento da concentração de clethodim, caracterizando melhores respostas entre as doses de 0,0300 e $0,0600 \mathrm{~kg} \mathrm{ha}^{-1}$. Para o sethoxydim, a estimativa do modelo demonstra melhor resposta para redução do número de inflorescências para concentração de aproximadamente $0,2500 \mathrm{~kg} \mathrm{ha}^{-1}$.

\section{CONCLUSÕES}

Os herbicidas clethodim e sethoxydim viabilizaram a redução da altura e do número de inflorescências da espécie Paspalum notatum, sendo o sethoxydim mais seletivo em relação à preservação das características visuais do gramado.

Tabela 3. Porcentagem de intoxicação na parte aérea do gramado de Paspalum notatum em resposta a aplicação de clethodim. (Experimento 1)

Table 3. Percentage of intoxication in aerial part of Paspalum notatum turfgrass in response to clethodim sprayer. (Experiment 1)

\begin{tabular}{|c|c|c|c|c|c|}
\hline \multirow[t]{2}{*}{ Tratamentos } & \multirow{2}{*}{$\frac{\text { Dosagens }}{\left(\mathrm{kg} \text { i.a. } \mathrm{ha}^{-1}\right)}$} & \multicolumn{4}{|c|}{ Intoxicação da parte aérea do gramado $(\%)$} \\
\hline & & $7 \mathrm{DAA}^{1}$ & $21 \mathrm{DAA}$ & 35 DAA & 49 DAA \\
\hline T1. clethodim ${ }^{2}$ & 0,060 & $27,3 \mathrm{a}$ & 49,0 a & 36,3 a & $4,5 \mathrm{a}$ \\
\hline T2. clethodim & 0,030 & $12,3 \mathrm{~b}$ & $30,0 \mathrm{~b}$ & $25,8 \mathrm{~b}$ & $3,3 \mathrm{a}$ \\
\hline T3. clethodim & 0,015 & $6,8 \mathrm{c}$ & $9,0 \mathrm{c}$ & $13,8 \mathrm{c}$ & $0,0 \mathrm{~b}$ \\
\hline T4. clethodim & 0,0075 & $3,5 \mathrm{~cd}$ & $2,8 \mathrm{~d}$ & $2,8 \mathrm{~d}$ & $0,0 \mathrm{~b}$ \\
\hline T5. clethodim & 0,00375 & 2,3 de & $2,0 \mathrm{~d}$ & $2,5 \mathrm{~d}$ & $0,0 \mathrm{~b}$ \\
\hline T6. testemunha & - & $0,0 \mathrm{e}$ & $0,0 \mathrm{~d}$ & $0,0 \mathrm{~d}$ & $0,0 \mathrm{~b}$ \\
\hline Fcalc & - & $179,94 *$ & $90,45^{*}$ & $66,86^{*}$ & $19,51 *$ \\
\hline C.V (\%) & - & 17,29 & 26,64 & 26,71 & 10,21 \\
\hline DMS $(5 \%)$ & - & 3,44 & 9,55 & 8,28 & 5,82 \\
\hline
\end{tabular}

${ }^{1}$ Dias após a aplicação dos tratamentos no gramado. ${ }^{2}$ Herbicida de nome comercial Select ${ }^{\circledR}$.

Valores seguidos de mesma letra na mesma coluna não diferem entre si pelo teste de Tukey ao nível de 5\% de probabilidade.

$*$ = Significativo a $5 \%$ de probabilidade; ${ }^{N S}=$ Não significativo. 
Tabela 4. Porcentagem de intoxicação na parte aérea do gramado de Paspalum notatum em resposta a aplicação de sethoxydim. (Experimento 2).

Table 4. Percentage of intoxication in aerial part of Paspalum notatum turfgrass in response to sethoxydim sprayer. (Experiment 2)

\begin{tabular}{lcccccc}
\hline Tratamentos & \multicolumn{2}{c}{ Dosagens } & & \multicolumn{4}{c}{ Intoxicacão da parte aérea do gramado (\%) } \\
\cline { 2 - 6 } \cline { 5 - 7 } & $\left(\mathrm{kg} \mathrm{i.a.} \mathrm{ha}^{-1}\right)$ & & $7 \mathrm{DAA}^{1}$ & $21 \mathrm{DAA}$ & $35 \mathrm{DAA}$ & $49 \mathrm{DAA}$ \\
\hline T1. sethoxydim & 0,2500 & & $6,0 \mathrm{a}$ & $18,3 \mathrm{a}$ & $11,3 \mathrm{a}$ & $5,0 \mathrm{a}$ \\
T2. sethoxydim & 0,1250 & & $2,3 \mathrm{~b}$ & $8,3 \mathrm{~b}$ & $6,0 \mathrm{~b}$ & $3,3 \mathrm{~b}$ \\
T3. sethoxydim & 0,0625 & & $0,0 \mathrm{c}$ & $3,0 \mathrm{c}$ & $3,0 \mathrm{bc}$ & $2,8 \mathrm{~b}$ \\
T4. sethoxydim & 0,03125 & & $0,0 \mathrm{c}$ & $3,0 \mathrm{c}$ & $3,0 \mathrm{bc}$ & $2,3 \mathrm{~b}$ \\
T5. sethoxydim & 0,01562 & $0,0 \mathrm{c}$ & $0,0 \mathrm{c}$ & $0,0 \mathrm{c}$ & $0,0 \mathrm{c}$ \\
T6. testemunha & - & $0,0 \mathrm{c}$ & $0,0 \mathrm{c}$ & $0,0 \mathrm{c}$ & $0,0 \mathrm{c}$ \\
\hline Fcalc & - & $71,92^{*}$ & $66,29 *$ & $41,82^{*}$ & $47,40^{*}$ \\
C.V (\%) & - & 41,81 & 31,62 & 33,95 & 25,59 \\
DMS $(5 \%)$ & - & 1,32 & 3,93 & 3,02 & 1,29 \\
\hline
\end{tabular}

${ }^{1}$ Dias após a aplicação dos tratamentos no gramado. ${ }^{2}$ Herbicida de nome comercial Poast ${ }^{\circledast}$.

Valores seguidos de mesma letra na mesma coluna não diferem entre si pelo teste de Tukey ao nível de 5\% de probabilidade.

$*$ = Significativo a $5 \%$ de probabilidade; ${ }^{\text {NS }}=$ Não significativo.

Tabela 5. Teor de clorofila nas folhas do gramado de Paspalum notatum em resposta a aplicação de clethodim. (Experimento 1) Table 5. Chlorophyll tenor in leaves of Paspalum notatum turfgrass in response to clethodim sprayer. (Experiment 1)

\begin{tabular}{|c|c|c|c|c|c|}
\hline \multirow[t]{2}{*}{ Tratamentos } & \multirow{2}{*}{$\frac{\text { Dosagens }}{\left(\text { kg i.a. ha }^{-1}\right)}$} & \multicolumn{4}{|c|}{ Teor de clorofila nas folhas $\left(\mu \mathrm{g} . \mathrm{dm}^{-2}\right)$} \\
\hline & & $7 \mathrm{DAA}^{1}$ & 21 DAA & 35 DAA & 49 DAA \\
\hline T1. clethodim ${ }^{2}$ & 0,060 & 30,2 & 30,5 & 31,6 & 33,1 \\
\hline T2. clethodim & 0,030 & 29,4 & 31,8 & 31,6 & 29,8 \\
\hline T3. clethodim & 0,015 & 29,8 & 30,8 & 31,6 & 29,1 \\
\hline T4. clethodim & 0,0075 & 32,1 & 32,1 & 28,9 & 29,5 \\
\hline T5. clethodim & 0,00375 & 31,2 & 29,8 & 29,6 & 29,1 \\
\hline T6. testemunha & - & 32,0 & 32,0 & 31,3 & 29,6 \\
\hline Fcalc & - & $1,80^{\mathrm{NS}}$ & $2,76^{\mathrm{NS}}$ & $1,37^{\mathrm{NS}}$ & $2,62^{\mathrm{NS}}$ \\
\hline C.V (\%) & - & 5,54 & 3,62 & 6,57 & 6,32 \\
\hline DMS (5\%) & - & 3,92 & 2,60 & 4,64 & 4,35 \\
\hline
\end{tabular}

${ }^{1}$ Dias após a aplicação dos tratamentos no gramado. ${ }^{2}$ Herbicida de nome comercial Select ${ }^{\circledast}$.

Valores seguidos de mesma letra na mesma coluna não diferem entre si pelo teste de Tukey ao nível de 5\% de probabilidade.

$*=$ Significativo a $5 \%$ de probabilidade; ${ }^{\text {Ns }}=$ Não significativo.

Tabela 6. Teor de clorofila nas folhas do gramado de Paspalum notatum em resposta a aplicação de sethoxydim.

Table 6. Chlorophyll tenor in leaves of Paspalum notatum turfgrass in response to sethoxydim sprayer. (Experiment 2)

\begin{tabular}{|c|c|c|c|c|c|}
\hline \multirow[t]{2}{*}{ Tratamentos } & \multirow{2}{*}{$\frac{\text { Dosagens }}{\left({\left.\text { kg i.a. } \text { ha }^{-1}\right)}\right.}$} & \multicolumn{4}{|c|}{ Teor de clorofila nas folhas $\left(\mu \mathrm{g} . \mathrm{dm}^{-2}\right)$} \\
\hline & & $7 \mathrm{DAA}^{1}$ & 21 DAA & 35 DAA & 49 DAA \\
\hline T1. sethoxydim ${ }^{2}$ & 0,2500 & 28,8 & 32,2 & 31,6 & 29,2 \\
\hline T2. sethoxydim & 0,1250 & 30,7 & 32,7 & 29,8 & 29,3 \\
\hline T3. sethoxydim & 0,0625 & 29,9 & 30,9 & 29,7 & 27,3 \\
\hline T4. sethoxydim & 0,03125 & 30,5 & 30,9 & 30,1 & 28,4 \\
\hline T5. sethoxydim & 0,01562 & 32,3 & 30,4 & 31,4 & 28,8 \\
\hline T6. testemunha & - & 32,0 & 32,0 & 31,3 & 29,6 \\
\hline Fcalc & - & $2,22^{\mathrm{NS}}$ & $1,09^{\mathrm{NS}}$ & $0,77^{\mathrm{NS}}$ & $1,19^{\mathrm{NS}}$ \\
\hline C.V (\%) & - & 5,71 & 5,58 & 6,39 & 5,09 \\
\hline DMS $(5 \%)$ & - & 4,03 & 4,04 & 4,49 & 3,36 \\
\hline
\end{tabular}

${ }^{1}$ Dias após a aplicação dos tratamentos no gramado. ${ }^{2}$ Herbicida de nome comercial Poast ${ }^{\circledast}$.

Valores seguidos de mesma letra na mesma coluna não diferem entre si pelo teste de Tukey ao nível de 5\% de probabilidade.

$*=$ Significativo a $5 \%$ de probabilidade; ${ }^{\text {NS }}=$ Não significativo. 


\section{REFERÊNCIAS}

ALCÂNTRA, P. B.; BUSFARAH, G. Plantas Forrageiras: gramíneas e leguminosas. São Paulo: Nobel, 1979. 150p.

ANGELIS NETO, G; ANGELIS, B. L. D. Plantas ornamentais: do paisagismo a outras aplicações. Revista Brasileira de Horticultura Ornamental, Campinas, v.5, n.1,p.12-19, 1999.

BARBOSA, J. G. et al. Plantio de gramados de Paspalum notatum Flügge "batatais": tipos de mudas e doses de fertilizantes. Revista Brasileira de Horticultura Ornamental, Campinas, v.3, n.2, p. 50-54, 1997.

COUSENS, R. Misinterpretations of results in weed research though inappropriate use of statistics. Weed Research, v.28, p.281-289, 1988.
DEMATTÊ, M. E. S. P. Aplicação de nitrogênio, fósforo, potássio, adubo orgânico e calcário dolomítico na produção de sementes de grama-batatais (Paspalum notatum Flügge) em Latossol Vermelho Escuro. 1983. 34p. Tese (Doutorado em Agronomia). Escola Superior de Agricultura “'Luiz de Queiróz", Piracicaba, 1983.

EUROPEAN WEED RESEARCH COUNCIL - EWRC. Methods in weeds research. Weed Research, Oxford, v.4, p.88. 1964.

FREITAS, F. C. L. et al. Influência de cimetacarb e clethodim como reguladores de crescimento em gramado. In: CONGRESSOBRASILEIRODACIÊNCIADAS PLANTAS DANINHAS, 21, 1997, Caxambu: Resumos... Caxambú: SBCPD, 1997.p.325.

GOATLEY, J. M.; MALDDOX, V. L.; WATKINS, R. M. Bahiagrass response to a plant growth regulator as effected by moving interval. Crop Science, v.38, n.1, p.196-200, 1998.
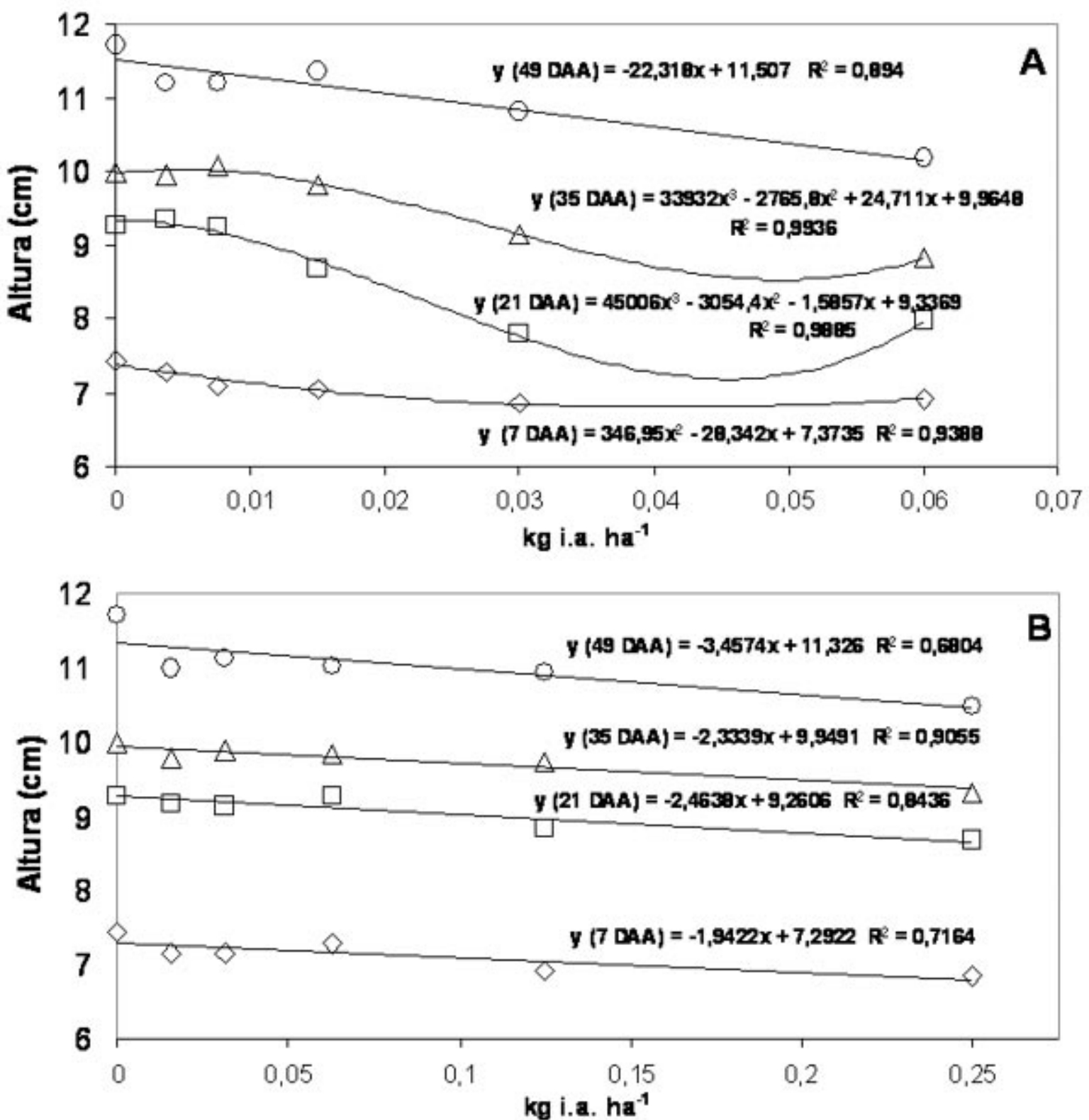

Figura 1. Altura do gramado de Paspalum notatum aos 7, 21, 35 e 49 DAA em resposta a aplicação de clethodim (A) e sethoxydim (B). Figure 1. Height of Paspalum notatum turfgrass submitted of clethodim (A) and sethoxydim (B) sprayer. 
IBGE - Cidades. Instituto Brasileiro de Geografia e Estatística. Coordenadas Geográficas das Cidades, 2004. Disponível em: <http://www.ibge.gov.br/home/presidencia/ noticias /notavisualiza.php?idnota $=3>$ Acesso em: 3 jan. 2004.

ITOGRASS. Guia de identificação de manejo de pragas e doenças em gramados. São Paulo: Textoart Gráfica e Editora Ltda, 2003. 20p.

JIANG, H.; FRY, J. Drought responses of perennial ryegrass treat plant growth regulators. Hortscience, v.33, n.2, p.270273, 1998.

JONHSON, B. J. Response of bahiagrass (Paspalum notatum) to plant growth regulators. Weed Technology, n. 4,p. 895-899, 1990.

JONHSON, B. J. influence of plant-growth regulators and mowing on to bahiagrasses. Agronomic Journal, v. 86, n. 5,p. 805-810, 1994.

KAUFMANN, J. E. Growth regulators for turf. Grounds Maintance, v.21, n.5, p.72, 1986.
KISSMANN, K. G. Plantas infestantes e nocivas. Tomo I. 2.ed. São Paulo: BASF, 1997. p.679-684.

LOPES, L. C. Anotações de aulas de jardinocultura. Viçosa: UFV, 1981. 8p. (mimiografado).

LORENZI, H. Plantas daninhas do Brasil: terrestres, aquáticas, parasitas e tóxicas. 3 ed. Nova Odessa: Plantarum, 2000.349p.

LORENZI, H.; SOUZA, H. M. Plantas Ornamentais do Brasil: arbustivas, herbáceas e trepadeiras. 3 ed. Nova Odessa: Plantarum, 2001. 558p.

PIMENTEL GOMES, F. A estatística moderna na pesquisa agropecuária. Piracicaba: POTAFOS, 1987. 160p.

QUIAN, Y. L.; ENGELKE, M. C. Influence of trinexapacethey on Diamond Zoysiagrass in a shade environment. Crop Science, v.39, p.2002-208, 1999.

RICHARDSON, M. D. Turf quality and freezing tolerance of Tifway bermudagrass as affected by late season nitrogen and trinexapac-ethyll. Crop Science, v.42, p.1612-1626, 2002.
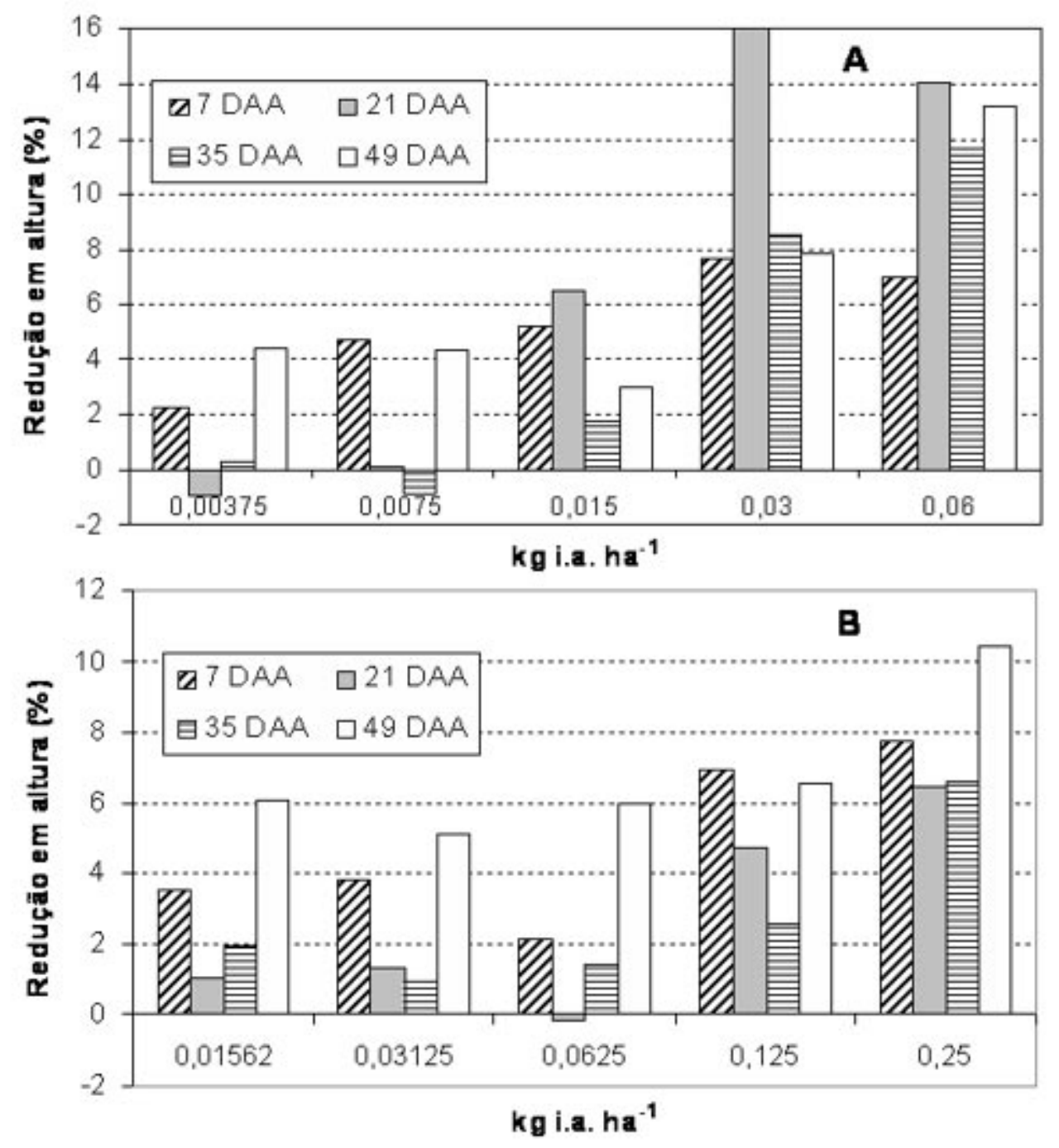

Figura 2. Redução percentual da altura do gramado de Paspalum notatum submetido a aplicação de clethodim (A) e sethoxydim (B) aos $7,14,21$ e 49 DAA.

Figure 2. Percentile reduction of height in Paspalum notatum turfgrass submitted of clethodim (A) and sethoxydim (B) sprayer. 
RODRIGUES, B. N.; ALMEIDA, F. S. Guia de Herbicidas. $5^{\mathrm{a}}$ ed. Londrina, Ed. dos autores. 2005. 592p.

RODRIGUES, J. D.; GODOY, L. J. G. de.; ONO, E. O. Reguladores Vegetais: bases e princípios para utilização em gramados. In:__org.). II SIGRA -Simpósio sobre Gramados - "Manejo de Gramas na Produção e em Gramados Formados". Botucatu: FEPAF, 2004. 10p.

SOCIEDADE BRASILEIRADACIÊNCIADAS PLANTAS DANINHAS. Procedimentos para instalação, avaliação e análise de experimentos com herbicidas. Londrina: SBCPD, 1995.42p.

TURFGRASS PRODUCERS INTERNATIONAL. Turfgrass - functional, recreational e aesthetic. Turf Resource Center, 2002. Disponível em: <http//www.turgrasssod.org/trc/ statistics.html (acesso em 23 Ago. 2002).
UNRUH, J. B. Biologia de Gramas de Estação Quente. In: (org.). II SIGRA - Simpósio sobre Gramados -

"Manejo de Gramas na Produção e em Gramados Formados". Botucatu: FEPAF, 2004. 31p.

VIDAL, R. A.; MEROTTO JÚNIOR, A. Herbicidologia. $1^{\text {a }}$ ed. Porto Alegre: Edição dos Autores, 2001, 152p.

WATSHCKE, T. L.; WEHNER, D. J, DUICH, J. M. Initial and residual effects of growth regulators on a PennstarFylking Kentucky bluegrass blend. In: Proceedings N.E. Weed Science Society, v.34 p. 382-388, 1977.

WSSA. Herbicide handbook. 7.ed. Champaign, WSSA. 352p. 1994.
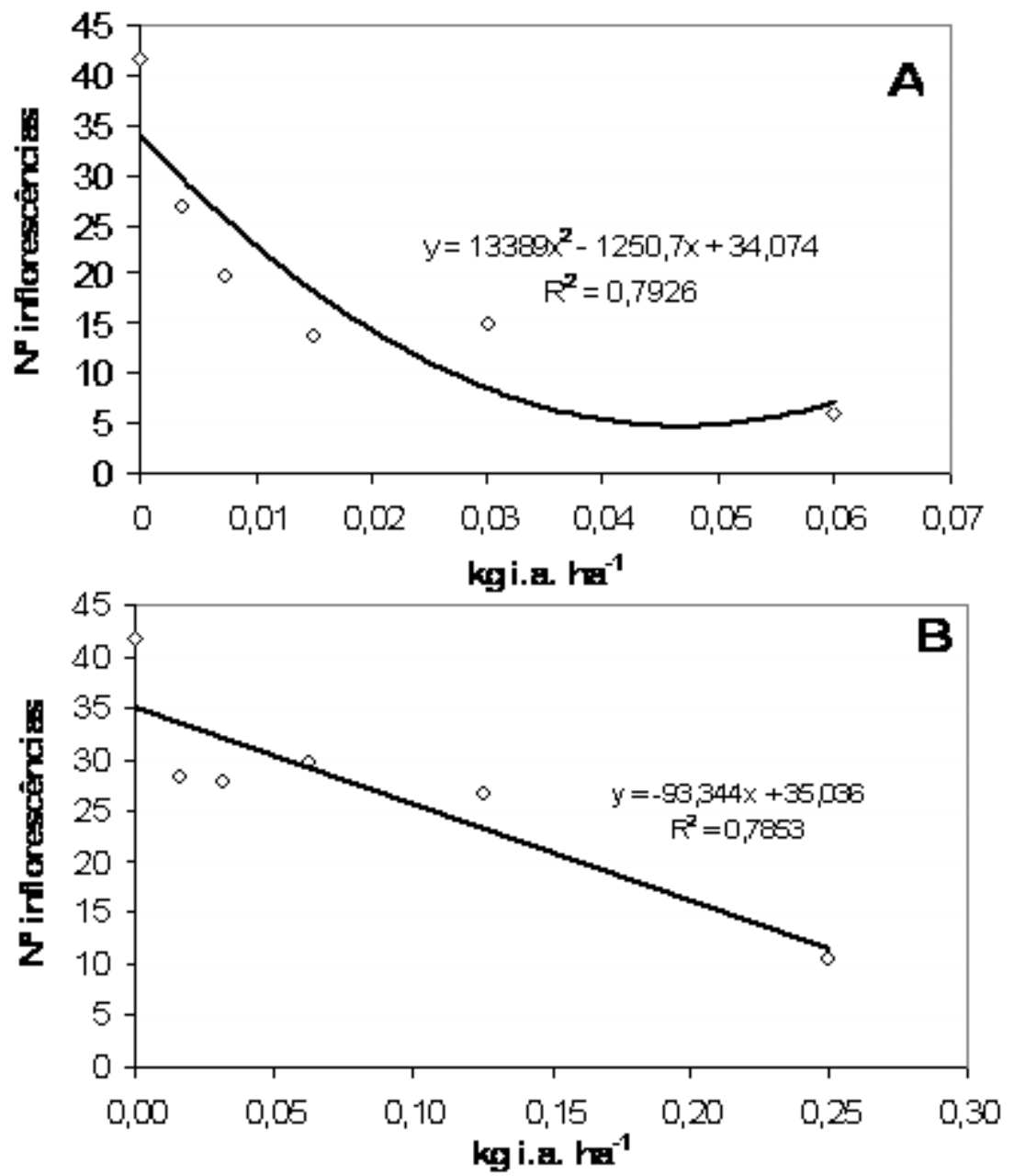

Figura 3. Número de inflorescências por $\mathrm{m}^{-2}$ de Paspalum notatum aos 80 DAA em resposta a aplicação de clethodim (A) e sethoxydim (B).

Figure 3. Number of Paspalum notatum inflorescences for $m^{-2}$ to 80 DAA in response of clethodim $(A)$ and sethoxydim $(B)$ sprayer. 